\title{
The Effect of Prophylactic Acupuncture Treatment in Women with Recurrent Cystitis: Kidney Patients Fare Better
}

\author{
TERJE ALRAEK, B.Ac., and ANDERS BAERHEIM, M.D., Ph.D.
}

\begin{abstract}
Objective: To evaluate how the effect of acupuncture treatment in recurrent cystitis relates to Traditional Chinese Medicine (TCM) diagnostic categories and physiologic measurements.

Setting/location: Two private acupuncture clinics in Bergen, Norway. Measurements were carried out in Haukeland University Hospital, Bergen.

Design and subjects: Ninety-eight (98) cystitis-prone women were randomly assigned to acupuncture treatment or no treatment. Main effect parameter was number of cystitis during 6 months observation time. Residual urine was measured with an automatic Bladder Scan 2500 (Diagnostic Ultrasound Corporation, Bothell, WA) at baseline, 2, 4 and 6 months.

Results: Twenty-two (22) patients had Spleen yang/qi $x u$ (Sp), 18 Kidney yang/qi $x u$ (Kid), and 18 Liver qi stagnation (Liv). Of the Kid group, 78\% were free of cystitis (irrespective of bacteriuria) during the observation period compared to $45 \%$ in the Sp group, $44 \%$ in the Liv group ( $p \leq$ 0.05 , Kid versus Sp and Liv), and 17\% in the nontreated group ( $p \leq 0.01$, versus all treated patients). One sixth as many episodes of acute cystitis occurred in the Kid group compared to the nontreated group (incidence rate ratio $[\mathrm{IRR}]=0.15,95 \%$ confidence interval $[\mathrm{CI}] 0.05-0.42, p \leq$ $0.001)$. Similarly, we found IRR $=0.49(0.25-0.83)$ in the Sp group and IRR $=0.42(0.21-0.80)$ in the Liv group. Subanalyzing for cystitis with detected bacteriuria yielded comparable results. Kid patients significantly reduced their residual urine from baseline to 6 months $(36.4 \mathrm{~mL}$ versus $12.9 \mathrm{~mL}, p \leq 0.05)$. The reduction was less and nonsignificant in Sp and Liv groups (31.5 $\mathrm{mL}$ versus $22.2 \mathrm{~mL}$, $36.4 \mathrm{~mL}$ versus $16.7 \mathrm{~mL}$, respectively). Residual urine remained unchanged among nontreated patients.

Conclusion: Our findings show a better acupuncture treatment effect on Kidney yang/qi $x u$ than on other TCM syndromes found in women with recurrent cystitis. This has practical implications, and highlights the need for considering different TCM diagnostic categories in acupuncture research.
\end{abstract}

\section{INTRODUCTION}

A cupuncture has been reported to have positive effects on several urologic disorders (Birch, 2001). Recently, two studies have shown acupuncture to reduce the recurrence rate in uncomplicated recurrent cystitis (Alraek et al., 2002; Aune et al., 1998). In the latter study our overall results showed that acupuncture reduced the recurrence rate to half the rate

Department of Public Health and Primary Health Care, Section for General Practice, University of Bergen, Bergen, Norway. 
among untreated women. The results from that study motivated us to elucidate whether the reported positive effect on the recurrence rate among cystitis-prone women was related to any specific Traditional Chinese Medicine (TCM) diagnostic category.

Cystitis-prone women must have some vulnerability behind their recurrent episodes of cystitis. The main syndromes found in TCM for the vulnerability by recurrent cystitis reflects disharmony in the following Zang/fu organs: Kidney, Spleen, Liver, Lungs, San Jiao and Urinary Bladder (Ross, 1984). Recently we have demonstrated in other material that 9 in 10 women with frequently recurrent cystitis fall into the three main and equally frequent TCM diagnostic categories of Kidney yang/qi $x u$, Spleen yang/qi $x u$, and Liver qi stagnation (Alraek et al., 2000). Diagnosing women prone for recurrent cystitis will focus on symptoms and signs in each individual patient, leading to a syndrome of disharmony. Treating such disharmonies is known as treating the root of the problem.

We have found that acupuncture reduces the incidence rate of cystitis in women prone to recurrent cystitis. The effect seems to correlate to a substantial reduction in the residual urine among the treated women (Alraek et al., 2002). In Western medicine, residual urine is a risk factor for recurrent cystitis in men with bladder outlet obstruction (prostatism). Residual urine is also recognized as a risk factor for recurrent cystitis both among healthy postmenopausal women (Raz, 2000) and in children (Hoebeke, 2001). Lack of $q i$ for expulsion of urine has been described in TCM literature, but without using residual urine as a concept for such consequences (Clavey, 1995). Retention of urine has been described in TCM, ranging from scanty and difficult urination to total absence of urination. If scanty with pain it is categorized under Painful-Urination-Syndrome (Lin in Chinese) and more related to the acute stages of cystitis (Maciocia, 1994), which are not addressed in the present study.

Based on this, the present study attempted to evaluate the effect of acupuncture treatment in recurrent cystitis as it relates to TCM diagnostic categories and physiologic measurements, among them residual urine.

\section{MATERIALS AND METHODS}

A total of 100 otherwise healthy cystitisprone women 18-60 years of age were recruited by advertising in local newspapers in Bergen, Norway. The inclusion process started in spring 1998 and lasted 12 months. Criteria for inclusion were three or more self-reported episodes of cystitis symptoms during the previous 12 months, provided that at least two of these had been diagnosed and treated as cystitis by a medical doctor. No antibiotics should have been taken during the previous three weeks, and women using cranberries as a prophylactic treatment were excluded. Subjects were excluded if they were pregnant or were known to have any complicating illness (e.g., diabetes, cancer, and obstruction of the urinary tract).

Two women failed to fulfill the inclusion criteria. The 98 women who were included were randomly assigned to acupuncture treatment or no treatment. The randomization ratio was 3:1 until the inclusion of patient 80 ; after this the ratio was $2: 1$ to give a necessary increase to the number patients in the control group. Two women randomly assigned to the control group withdrew because of disappointment of not being treated. In the acupuncture group, two women could not follow the treatment schedule, and were therefore also excluded. The study thus consists of 94 women: 67 in the acupuncture group and 27 in the no-treatment group.

Patients who left the study before the start of the observation period were regarded as dropouts. Patients who withdrew from study during the 6-month observation period were kept in the study with the actual observation time. Patients who became pregnant were excluded from the study at the time their pregnancies were diagnosed.

Inclusion processes were performed in a hospital setting, and included a written, informed consent, instruction in the use of a dipslide (Uricult ${ }^{\circledR}$, Life Sign, Someset, NJ) and baseline measurements, including skin conduction (sympathetic nerve activity) and residual urine measured by the automatic Bladder Scan 2500 (Diagnostic Ultrasound Corporation, Bothell, WA). A background questionnaire and 
a TCM symptom questionnaire (Alraek et al., 2000) were completed. The measurements were repeated at 2, 4, and 6 months and were performed by a specially trained nurse, not involved in any other part of the study. The measurements at 2 months for the treated participants were carried out within 2 weeks after the final treatment.

The TCM consultations were conducted in a private acupuncture practice. The diagnostic procedure included a clinical interview, tongue, and pulse diagnosis. Afterwards a closed envelope was opened, containing the patients' randomization to treatment or no treatment, which also specified which of two acupuncturists should perform the treatment.

Two acupuncturists (N.C. and T.A.) were present, working together toward an agreement on the correct diagnosis of the patient. Any disagreement was resolved by consensus. Patients were treated twice weekly for 4 weeks, in the patients' healthy phase between attacks of cystitis. The needles (Shao Long, China $0.30 \times 20 / 40 / 50 \mathrm{~mm}$ ) were inserted and de qi was obtained. Needles were manipulated after the arrival of $q i$ at insertion, one time during the session and at withdrawal of the needles. Manipulation was a combination of rotation with lifting and thrusting when appropriate. The needles were left in situ for at least 20 minutes, but for a maximum of 30 minutes. Needles were inserted in bladder points on the back (i.e., BL23 and 28) and stimulated until de qi was achieved, then withdrawn after manipulation according to TCM diagnosis. Several pathologic factors (TCM) are given in urologic problems. They can be present in shi or $x u$ conditions or a mixture of both. Moreover, chronic problems seem to occur on a background of $x u$ conditions especially of the Kidneys and Spleen (Maciocia, 1994). These theories and our clinical experience influenced our selection of acupuncture points. The most frequently used acu points were in Kid patients BL23, KI3, CV3, SP6, and BL28; among Spleen patients SP6, CV3, BL28, ST36, and SP9; and for Liver patients LR3, CV3, BL28, SP9 and SP6. During treatment the women were lying quietly. Prophylactics such as cranberries were not allowed to be used during the study. The two acupuncturists were graduates of different acupuncture schools: N.C. from Norsk Akupunkturskole (N.A.), Oslo, Norway, and T.A. from The International College of Oriental Medicine, EastGrinstead, England. Both had worked as acupuncturists for more than 15 years in separate clinical practices. One of the authors (T.A.) finished a $1 \frac{1}{2} 2$-year training in Chinese herbal medicine (N.A., Oslo 1998) and has been involved in research on acupuncture and recurrent cystitis since 1994.

The main effect measure was the number of acute cystitis during a 6-month follow-up period. Subjects were asked to seek medical aid in case of distal urinary symptoms. If a doctor was not available they were instructed to culture a preaddressed dip-slide and send it by mail to the laboratory for bacteriologic examination. Having completed this, the patients could start medical treatment according to their preference.

If symptoms of an acute cystitis were present at the day of treatment, this was taken into consideration and was treated according to TCM theory. If the patients were taking antibiotics, further treatment was postponed until they finished the antibiotic course (3-5 days).

Distal urinary symptoms were defined as dysuria, urinary frequency, and/or suprapubic discomfort. To analyze impact from the patients' view, and to be in agreement with recent understandings of the diagnosis of a cystitis, an acute cystitis was defined as acute distal urinary symptoms only, as urinalysis and culture are unnecessary for the diagnosis (Bent et al., 2002). Main results were subanalyzed for patients with bacteriuria $\left(10^{5}\right.$ or more colonyforming units per milliliter of uropathogens, or any amount of Staphylococcus saprophyticus). Samples for bacteriologic examination were collected from clean-voided urine using a dipslide (Uricult ${ }^{\circledR}$ ). The specimens were examined at the Department of Microbiology and Immunology, Haukeland University Hospital, using standard techniques.

The Regional Ethical Committee and the Norwegian Data Inspectorate approved the study.

\section{Data analysis}

The incidences of cystitis in the observation period were calculated as incidence rates (num- 
ber of cystitis in the 6 months observation period per-person half-year), and comparison between groups as incidence rate ratio (IRR) with 95\% confidence interval (CI). $P$ values for IRR were described by calculating CI for increasing significant levels (not shown). Differences between groups were for categorical data tested using Fisher exact test and $\chi^{2}$ tests, and for parametric data using independent $t$ test or analysis of variance (ANOVA). Differences within groups were tested using paired $t$ test. By subgroup analysis borderline significance are also presented.

\section{RESULTS}

A total of 94 cystitis-prone women 18 to 60 years of age were included. Of these, 67 women were randomly assigned to the treatment group and 27 to the no-treatment group. Six (6) were later excluded from the treatment group because of missing information and these were regarded as dropouts. Main diagnostic categories are given in Table 1. Six (6) of the 94 women were diagnosed into other diagnostic categories, respectively, 4 with Kidney yin $x u$, 1 with blood $x u$, and 1 with typical signs and symptoms of a damp heat condition in lower jiao. These 6 patients, 3 in the treated and 3 in the nontreated group, are excluded from the following data analysis because of small numbers.

Background data from the remaining 58 treated and 24 nontreated patients are given in Table 1 . There were no clinically significant dif- ferences between the groups in regard to background data.

Patients were diagnosed into three equally large TCM diagnostic categories (Table 1). In the treatment group, 22 women had Spleen yang/qi xu (38\%, 95\% CI 27-53\%), 18 had Kid yang/qi xu $(31 \%, 19-44 \%)$, and 18 had Liver $q i$ stagnation $(31 \%, 19-44 \%)$. Among the 24 patients in the control group, 10 patients $(42 \%)$ had Spleen yang/qi $x u, 9$ patients $(38 \%)$ had Kidney yang/qi $x u$, and 5 patients (21\%) had Liver qi stagnation.

Of all the treated patients (Table 2), significantly more women (78\%) in the Kidney yang/qi $x u$ group were free of cystitis during the 6month observation period than in the Spleen yang/qi $x u$ group (45\%) and the Liver qi stagnation group $(44 \%, p \leq 0.05)$. In the nontreated group, only $17 \%$ of the women were free of symptomatic cystitis during the observation period ( $p \leq 0.01$ versus all treated patients).

As Table 2 shows, acupuncture treatment in Kidney patients reduced the incidence rate to one sixth of the incidence rate in the nontreated group $(\mathrm{IRR}=0.15,95 \%$ C.I. $0.05-0.42, p \leq 0.001)$. The reduction versus the nontreated group was substantially less among Spleen $(\mathrm{IRR}=0.49$, $0.25-0.83, p \leq 0.05)$ and Liver $(\mathrm{IRR}=0.42$, $0.21-0.80, p \leq 0.05)$ patients. There were one third as many episodes of acute cystitis per-person-half-year in the Kidney yang/qi group than in the Spleen yang/qi and Liver $q i$ stagnation group (IRR $=0.35,0.12-1.00, p \leq 0.05)$.

When subanalyzing for cystitis with detected bacteriuria, there were one fourth as many

Table 1. Fifty-Eight Women with Uncomplicated Recurrent Cystitis: Acupuncture Syndromes and Background Data

\begin{tabular}{|c|c|c|c|c|}
\hline & $\begin{array}{c}\text { Spleen } \\
\text { qi/yang xu }\end{array}$ & $\begin{array}{c}\text { Kidney } \\
\text { qi/yang xu }\end{array}$ & $\begin{array}{l}\text { Liver qi } \\
\text { stagnation }\end{array}$ & $\begin{array}{c}\text { No } \\
\text { treatment }\end{array}$ \\
\hline Number of patients & 22 & 18 & 18 & 24 \\
\hline UTIs last 12 months & 6.1 & 6.5 & 5.6 & 4.9 \\
\hline Mean age & 39.7 & 40.2 & 32.7 & 39.1 \\
\hline Mean body mass index & $23.9^{\mathrm{a}}$ & 22.7 & 21.3 & 22.3 \\
\hline Mean age at first UTI & 16.7 & 14.5 & 12.4 & 14.0 \\
\hline Mean weeks since last UTI & 3.4 & 4.6 & 8.2 & 6.3 \\
\hline $\begin{array}{l}\text { Mean weekly number of } \\
\text { coitus previous month }\end{array}$ & 2.0 & 1.2 & 2.3 & 1.7 \\
\hline
\end{tabular}

a $p \leq 0.05$ vs. the other groups, ANOVA test.

UTIs, urinary tract infections; ANOVA, analysis of variance. 
Table 2. Episodes of Cystitis During a Six-Month Observation Period in Fifty-Eight Women Receiving tCM Acupuncture for Prophylaxis and for Twenty-Four Women Receiving No Treatment

\begin{tabular}{|c|c|c|c|c|}
\hline Number of patients & $\begin{array}{c}\text { Spleen } \\
\text { qi/yang xu } \\
\qquad 22\end{array}$ & $\begin{array}{c}\text { Kidney } \\
\text { qi/yang xu } \\
18\end{array}$ & $\begin{array}{c}\text { Liver qi } \\
\text { stagnation } \\
18\end{array}$ & $\begin{array}{c}\text { No } \\
\text { treatment } \\
24\end{array}$ \\
\hline \multicolumn{5}{|l|}{ Episodes of cystitis, any case } \\
\hline 0 & 10 & $14^{\mathrm{a}}$ & 8 & $4^{\mathrm{b}}$ \\
\hline 1 & 9 & 4 & 8 & 10 \\
\hline 2 & 3 & 0 & 2 & 4 \\
\hline 3 & 0 & 0 & 0 & 5 \\
\hline 5 & 0 & 0 & 0 & 1 \\
\hline Observation period in months & 5.4 & 5.5 & 6.0 & 6.2 \\
\hline IR & 0.76 & 0.24 & 0.66 & 1.55 \\
\hline IRR each against no treatment & 0.49 & $0.15^{\mathrm{c}}$ & 0.42 & \\
\hline $95 \%$ C.I. & $0.25-0.83$ & $0.05-0.42$ & $0.21-0.80$ & \\
\hline \multicolumn{5}{|c|}{ Episodes of cystitis with bacteriuria } \\
\hline 0 & 15 & 16 & 11 & 12 \\
\hline 1 & 7 & 2 & 7 & 7 \\
\hline 2 & 1 & 0 & 0 & 5 \\
\hline IR & 0.45 & 0.12 & 0.39 & 0.69 \\
\hline IRR each against no treatment & 0.65 & $0.17^{\mathrm{a}}$ & 0.57 & \\
\hline $95 \%$ C.I. & $0.28-1.39$ & $0.04-0.74$ & $0.24-1.37$ & \\
\hline
\end{tabular}

Results are presented as incidence rate (IR) calculated as number cystitis/person $\bullet$ half year. Differences between groups are shown using incidence rate ratio (IRR) with 95\% confidence intervals (CI).

${ }^{\mathrm{a}} p \leq 0.05$ kidney vs. spleen and liver, $\chi^{2}$ test.

$\mathrm{b}_{p} \leq 0.01$ all treated vs. no treatment, $\chi^{2}$ test.

$c_{p} \leq 0.001$ Kidney vs. no treatment, see Data analysis.

$\mathrm{IRR}=$ e.g., $\mathrm{IR}_{\text {Spleen }} / \mathrm{IR}_{\text {no treatment }} ; \mathrm{TCM}$, Traditional Chinese Medicine.

episodes in the Kidney yang/qi group than in the no-treatment group $(\mathrm{IRR}=0.17,0.04-0.74$, $p \leq 0.05)$. Comparing Spleen (IRR $=0.65,0.28$ 1.39) and Liver (IRR $=0.57,0.24-1.37$ ) groups with the nontreated group revealed no statistical significance.

Residual urine related to the TCM diagnostic categories are shown in Table 3. All treated women reduced their mean residual urine after 6 months compared to the nontreated women. However, residual urine in patients with Kidney yang/qi $x u$ was substantially more reduced at 6 months than in Spleen and Liver patients, reaching the level of one third of the baseline value ( $p \leq 0.05$ versus baseline). At 2 months control (just after treatment) there was an increase in the mean residual urine among the Spleen $y a n g / q i$ group compared to the two other treated groups ( $p \leq 0.05$, ANOVA test).

The patients diagnosed as having a Liver $q i$ stagnation measured at inclusion highest on the sympathicus score 10.9 versus 9.6 for Spleen and 9.5 for Kidney $(p=0.13)$. After 6 months there were an overall reduction in sympathicus among treated subjects, but without any difference between groups (Liver, 7.1; Spleen, 7.3; and Kidney, 6.9).

The results of the self-administered symptom questionnaire filled in before inclusion and TCM diagnosing are shown in Table 4. Loose stools, anxiety, depression, and cold hands and feet were substantially more present in the Kidney yang/qi $x u$ group than in the two other groups. Preferences for cold drinks were less in the Kidney group compared to the two others. Being easily angered was more present among patients in the Liver $q i$ stagnation group $(p=$ 0.13 ) compared to the Kidney and Spleen groups. There were no statistical differences in the other questions.

At baseline, patients in the Kidney yang/qi group reported more problems on starting urination $(p=0.10)$, and more need for using extra pressure to perform micturation ( $p \leq$ 0.01 ) than did the Spleen and Liver patients (Table 4). 
Table 3. Residual Urine in Milliliters in Fifty-Eight Women with Uncomplicated Recurent Cystitis Treated with Acupuncture, Related to TCM Syndromes

\begin{tabular}{lcccc}
\hline & $\begin{array}{c}\text { Spleen } \\
\text { qi/yang } \mathrm{xu}\end{array}$ & $\begin{array}{c}\text { Kidney } \\
\text { qi/yang } \mathrm{xu}\end{array}$ & $\begin{array}{c}\text { Liver qi } \\
\text { stagn }\end{array}$ & $\begin{array}{c}\text { No } \\
\text { treatment }\end{array}$ \\
\hline Baseline & 31.5 & 36.4 & 36.4 & 32.8 \\
2 months & $52.0^{\mathrm{a}}$ & 22.4 & 18.6 & 29.2 \\
4 months & 29.7 & 21.7 & 13.9 & 31.8 \\
months & 22.2 & $12.9^{\mathrm{b}}$ & 16.7 & 35.1 \\
\hline
\end{tabular}

${ }^{a} p \leq 0.05$ comparing groups, ANOVA test.

$\mathrm{b}_{p} \leq 0.05$ vs. baseline, using paired $t$ test.

Data on no-treatment group for comparison. TCM, Traditional Chinese Medicine.

\section{DISCUSSION}

Among the three equally large groups of cystitis-prone women diagnosed respectively as Spleen qi/yang $x u$, Kidney qi/yang $x u$, and Liver $q i$ stagnation, only patients diagnosed as having Kidney qi/yang $x u$ benefited significantly from the acupuncture treatment. The treatment effect was paralleled by a substantially greater reduction in the residual urine in the Kidney yang/qi xu patients than in the other treated patients.
In an earlier, comparable study on recurrent cystitis (Aune et al., 1998) a group treated with (invasive) sham acupuncture had an incidence rate $(I R)=0.59$ compared to no treatment. This is not different from the Spleen (and Liver) patients in the present study, when we adjusted for the differences in cystitis rate in the two materials. Individual (TCM) acupuncture treatment of cystitis-prone women with the syndromes of Spleen qi/yang $x u$ (and Liver qi stagnation) may in other words not be any more effective than shallow needling outside

Table 4. TCM Symptoms among Fifty-Eight Women with Uncomplicated Recurent Cystitis in the Three Main Diagnostic Categories

\begin{tabular}{lccc}
\hline & Spleen yang/qi xu & Kidney yang/qi xu & Liver qi stagnation \\
\hline Loose stools & $2 / 21$ & $7 / 17^{\mathrm{a}}$ & $2 / 18$ \\
Anxiety & $8 / 22$ & $12 / 16^{\mathrm{a}}$ & $5 / 18$ \\
Depression & $4 / 22$ & $7 / 17^{\mathrm{b}}$ & $1 / 18$ \\
Angry & $4 / 22$ & $4 / 16$ & $7 / 17^{\mathrm{c}}$ \\
Feeling cold on hands and feet & $13 / 22$ & $15 / 17^{\mathrm{d}}$ & $12 / 18$ \\
Preference for cold drinks & $13 / 22$ & $6 / 16^{\mathrm{e}}$ & $10 / 16$ \\
Tiredness & $11 / 22$ & $10 / 17$ & $7 / 18$ \\
Regular eating habits & $19 / 22$ & $14 / 17$ & $14 / 18$ \\
Regular stools & $15 / 21$ & $14 / 17$ & $14 / 18$ \\
Nightsweat & $5 / 22$ & $5 / 17$ & $4 / 18$ \\
Extra pressure for micturation & $4 / 22$ & $10 / 18^{\mathrm{a}}$ & $5 / 18^{\mathrm{f}}$ \\
Problems on starting urination & $2 / 22$ & & $4 / 18$ \\
Vaginal discharge: & & $10 / 16$ & $1 / 18$ \\
$\quad$ White & $9 / 21$ & $1 / 16$ & $8 / 18$ \\
Yellow & $6 / 21$ & $4 / 16$ & $3 / 18$ \\
No special color & $4 / 21$ & $7 / 18$ \\
\hline
\end{tabular}

Symptoms were recorded from a TCM questionnaire introduced before the TCM clinical diagnostic procedures.

${ }^{a} p \leq 0.01$, kidney vs. two other groups.

$\mathrm{b}_{p} \leq 0.05$, kidney vs. two other groups.

$c_{p} p=0.13$, liver vs. two other groups.

$\mathrm{d}_{p}=0.11$, kidney vs. two other groups.

$\mathrm{e}_{p}=0.13$, kidney vs. two other groups.

$\mathrm{f}_{p}=0.10$, kidney vs. two other groups.

All calculated by Fisher's exact test.

TCM, Traditional Chinese Medicine. 
known acupuncture points (invasive sham acupuncture).

According to TCM, the Kidneys govern the water metabolism. They act as a gate that opens and closes to regulate the flow of Body Fluids. Normally the opening and closing functions are intact and the Bladder will receive $q i$ from the Kidney for transportation and excretion of fluids. Lack of yang or $q i$ in the Kidneys may give rise to symptoms such as incontinence, enuresis and lack of power for urine excretion (Clavey, 1995). At baseline in this study, Kidney yang/qi patients reported significantly more often than the other diagnostic groups to have problems starting urination and that they had to use extra pressure to perform micturation. These problems may have changed due to a treatment-induced increase of Kidney yang qi, which is the preeminent $q i$ for expulsion of urine from the Bladder. Normalization of the voiding process is also the goal for bladder training, most often used in children with unstable bladder (Hellstrøm et al., 1987). This training is expected to reduce residual urine and thereby the recurrence rate of cystitis (Koff and Murtagh, 1983). We found parallel changes in our material, with a statistical reduction in the residual urine among the treated patients with a Kidney yang/qi xu. A possible interpretation is that treatment-induced increase of yang in Kidney yang/qi $x u$ patients is hitting "the root of the problem." Increased yang qi may normalize bladder motility and reduce residual urine, hence accounting for the treatment results in the Kidney group.

The Liver in TCM is responsible for the free flow of $q i$ in the lower jiao; the Liver meridian has an intersection at CV3 with the urinary bladder. Hence, Liver qi stagnation can lead to heat or damp, giving rise to different urinary symptoms (e.g., urinary obstruction and urinary frequency). It is typical that these symptoms are worsened by stress and emotional tension (Clavey, 1995). In a clinical interview, such information, together with a wiry pulse and signs of Liver qi stagnation on the tongue, build the basis for a Liver qi stagnation diagnosis. In our study, anger was the one parameter in which patients with Liver qi stagnation differed from the other diagnostic groups.
We found a high sympaticus score at baseline among Liver patients. This high score may be the result of the tension, anger, frustration, irritability, and wiry pulse, which according to TCM is characteristic of a Liver qi stagnation. Adrenergic tension may inhibit the activity in the parasympathetic nerve system, increasing the tonus in the internal sphincter of the bladder, and reducing the tonus in the detrusor muscles. These combined effects may hinder complete bladder emptying, causing residual urine.* The women treated as having Liver qi stagnation showed an immediate response in the residual urine after treatment. This may reflect a return of "free flow" of Liver qi in these patients. Still, our results were not very promising for these patients. Having no prior knowledge of the present results, we treated the exact pattern generated at the moment by the particular configuration of manifesting symptoms and signs in all patients as described by Kaptchuk in 1983. This was not sufficient. Maybe patients with Liver qi stagnation need follow-up treatments, mainly because of the strong impact emotions seem to have on their qi circulation.

The women diagnosed as having Spleen qi/yang $x u$ had the highest mean body-mass index (BMI) compared to the two other groups. This may be looked on as a manifestation of a slow metabolism or a lack of transformation and transportation function of the Spleen yang $q i$. The Spleen is especially sensitive to dampness; lack of Spleen yang qi will allow the dampness to grow even stronger and develop into a persistent condition. Spleen patients did not fare well in our study. The women diagnosed and treated as Spleen yang/qi went through an increase of residual urine just after treatment, quite different from the results in the Kidney and the Liver group. Can the increase of residual urine be related to wrong treatment or is it a side-effect in some patients related to the use of certain acupuncture points? From our work, we cannot tell. Diuretic herbs (used carefully, according to TCM) given to relieve the dampness and, added to these, other herbs that could invigorate the transformation action of the

*Baerheim A. Lower Urinary Tract Infections in Women. Aspects of Pathogenesis and Diagnosis [thesis]. University of Bergen, Bergen, 1994. 
Spleen, may be a more appropriate treatment for group of patients with recurrent cystitis. This raises many questions, which warrants further studies.

The present study had an open design, because blinding is difficult for a manual treatment such as acupuncture. We allocated the control group to no treatment, because this is relevant for clinical practice and because our earlier study had shown a substantially higher effect of TCM acupuncture than invasive sham treatment (Aune et al., 1998). We therefore decided on a study comparing acupuncture treatment to no treatment, and instead relying on effect parameter that was blinded on several levels throughout the study.

Patients were recruited by advertisement, which may have selected respondents interested in acupuncture. However, this may not account for the differences in effect between the groups. Furthermore, we had an untreated control group, and used a clinical definition of cystitis, hence our data may be fairly valid for how acupuncture treatment works compared to no treatment in daily practice.

In conclusion, our findings show a better acupuncture treatment effect on Kidney yang/qi $x u$ than on other TCM syndromes found in women with recurrent cystitis. This has practical implications, and highlights the need for considering different TCM diagnostic categories in acupuncture research. Furthermore, a possible prophylactic treatment (TCM) scenario for cystitis-prone women might be: acupuncture for women diagnosed as having Kidney problems, Chinese herbal treatment for those diagnosed with Spleen problems, and after initial acupuncture, in Liver patients they may further benefit from mind-body therapies with focus on relaxation and stress management. Further studies are warranted to answer these issues.

\section{ACKNOWLEDGMENTS}

We thank the Norwegian Research Council and the Eckbos Legacy for funding the study. We also thank Norbert Cools, the participating acupuncturist, and Gerd Anne Wincentsen, the nurse who carried out all physical measurements.

\section{REFERENCES}

Alraek T, Soedal FIL, Fagerheim US, Digranes A, Baerheim A. Acupuncture treatment in the prevention of uncomplicated recurrent lower urinary tract infections in adult women. Am J Public Health 2002;92:1609-1611.

Alraek T, Aune A, Baerheim. Traditional Chinese medicine syndromes in women with frequently recurring cystitis: Frequencies of syndromes and symptoms. Complement Ther Med 2000;8:260-265.

Aune A, Alraek T, Huo Lihua, Baerheim A. Acupuncture in the prophylaxis of recurrent lower urinary tract infection in adult women. Scand J Prim Health Care 1998;16:37-39.

Bent S, Nallamothu KB, Simel LD, Fihn DS, Saint S. Does this woman have an acute uncomplicated urinary tract infection? JAMA 2002;287:2701-2710.

Birch S. An overview of acupuncture in the treatment on stroke, addiction, and other health problems. In: Stux G, Hammerschlag R, eds. Clinical Acupuncture. Scientific Basis. Berlin: Springer, 2001:131-149.

Clavey S. Fluid Physiology and Pathology in Traditional Chinese Medicine. Melbourne: Churchill Livingstone, 1995.

Hellstrøm AL, Hjalmås K, Jodal U. Rehabilitation of dysfunctional bladder in children: Method and 3-year follow up. J Urol 1987;138:847-848.

Hoebeke P, Van Laecke E, Van Camp C, Raes A, Van De Walle J. One thousand video-urodynamic studies in children with non-neurogenic bladder sphincter dysfunction. BJU Int 2001;87:575-580.

Kaptchuk T. Chinese Medicine. The Web That has no Weaver. London: Rider, 1983.

Koff SA, Murtagh DS. The uninhibited bladder in children: Effect of treatment on recurrence of urinary infection and on vesicoureteral reflux resolution. J Urol 1983;130:1138-1140.

Maciocia G. The Practice of Chinese Medicine. The Treatment of Diseases with Acupuncture and Chinese Herbs. London: Churchill Livingstone, 1994.

Raz R, Gennesin Y, Wasser J, et al. Recurrent urinary tract infections in postmenopausal women. Clin Infect Dis 2000;30:152-156.

Ross J. Zang Fu. The Organ Systems of Traditional Chinese Medicine. Bristol: Presto Print, 1984.

Address reprint requests to: Terje Alraek, B.Ac. Department of Public Health and Primary Health Care Section for General Practice University of Bergen

Ulriksdal 8C Bergen N-5009 Norway

E-mail: terje.alraek@isf.uib.no 